\title{
DEFINING THE TERMS OF WATER DISTRIBUTION IN TEXTILE BY THE PHOTOMETRY METHODS
}

\author{
Ganna Shchutska \\ Higher State Educational Establishment "Kyiv College of Light Industry" \\ 29 I. Kudri str., Kyiv, Ukraine, 01042 \\ polischuka.kklp@gmail.com
}

\begin{abstract}
The work proved computer photometric method for determining the water concentration in the line textile samples. The aim of the article is the testing of computer visualization techniques to determine the sorption characteristics of textile structural components. The main result is the determination of the actual empirical distribution functions in linear liquid samples. For this purpose one of the boundaries of the test material was set in contact with coloured water. Thanks to the diffusion properties of the material, water spread along the sample changing its brightness. Using of visualization enabled to determine the concentration in the textile sample. Experimental regressive dependence of concentration in a sample of different factors was developed. The proposed concentration dependence of the water from the coordinates and time has the form of exponential function. Exponent index is a characteristic feature of this material, which is characterized its absorption properties. Constants that describe the intensity of a linear sorption of the sample of material were defined. The results can be used in predicting of the water distribution and process modeling of discrete material structure.
\end{abstract}

Keywords: photometric method, textile samples, medical textiles, wound dressings, fibres, cotton, yarn.

\section{Introduction}

One of the most important indicators of textile materials is their sorption and desorption properties. Researched data show fairly deep results of definition of similar characteristics for both natural and synthetic materials. It should be noted that these data are derived largely as a result of experiments for material as general. Several sources suggest taking into account structural details in determining the overall characteristics of the material. It is desirable for these models to determine the sorption characteristics of individual threads. Existing standards do not provide methods for such tests. Due to advances in computer imaging, photometry methods are proposed to use for this purpose.

So there is a contradiction between the need to simulate absorption characteristics in textile materials, not elaborated methods to determine these characteristics for individual structural elements and opportunities of modern methods of computer visualization.

The aim of this article is testing of computer visualization techniques to determine the sorption characteristics of textile structural components.

Problems of moisture distribution in the textile materials are actual in the design and operation of medical textiles and wound dressings. It is important to predict the time of water passage through the material. Also, the knowledge of the water concentration over time is important.

\section{Overview of the problem}

Materials of different structures and compositions were investigated in a number of publications. In particular, [1] noted that cotton is a hygroscopic material, which gives uncomfortable feeling when moistened. For synthetic materials such as nylon or polyester characteristic properties allows more moisture to remove them. Other materials, such as cotton acrylic [2] or a mixture of cotton with polyester components [3] studied on sorption taking into account capillary effects. Managing the process of passing the moisture in the cotton material studied in [4].

Separately the working conditions of moist textiles and impact properties of blends of yarn were investigated [5]. It is shown that textile products made of a mixture of polyester and cotton fibers provide the best feeling when moistened [6].

Some articles also investigated the mutual influence of moisture and vapor passage through textiles [7]. The influence of finishing on the diffusion properties of the tissue studied in [8]. 
There is a linear process of passing the water through the textile material, impact of water dynamics in the material in its passage [9]. This fact requires developing of very complex models that cannot be accounted for by existing methods [10].

Attempt of developing a continuum model taking into account the process of water accumulation in the material is presented in [11]. Unfortunately, this model requires microscopic material constants, determination methods are not shown.

In [12] special ball textiles, consisting of simple interweaving fabrics and microporous films were studied. According to the research results from hydraulic resistance, moisture conservation was obtained.

It should be noted that in all these cases, the material is studied as a whole. In constructing experiments analysis of its structural characteristics must be done.

In a number of articles influence of real structure for moisture transfer is marked [13, 14$]$.

The article [15] made attempt to take into account the structural characteristics of textiles in determining the characteristics of moisture absorption.

For this purpose, [16] high-density tissue were analyzed. The structure of the material, which directly affects properties such as passing and retaining moisture, was taken into account. During the analysis physical and optical research methods were used. Also data on the generation of three-dimensional virtual tissue was presented [17].

Attempt of process simulation of passing the water through a fabric structure was made in [18]. Application of these methods for three-dimensional case is given in [19]. The downside of recent studies is that they must rely on experimental data for individual structural elements that are generally unknown. At the same time if the definition this data is done, methods [18, 19] can be used in universal situations. We propose to use photometric method for determining the water concentration in the line samples of textile materials, given the increasing possibilities of digital technology for measuring visual performance of materials. It is obvious that in the current proposals $[20,21]$ these methods are not widely implementing.

\section{Materials and methods}

The aim of the study is to determine the dynamic function of dependence of water concentration in the textile material, which can be used to predict its performance and durability.

To determine the parameters of the water distribution in linear samples complex experiments to determine the dynamics of change in concentration was carried out. It should be noted that it is difficult to determine the concentration in a direct way. Therefore, the method of changing the water brightness was used.

One of the boundaries of the test material was set in contact with colour water. Each textile material has its own diffusion properties. So, water spreads along the sample, changing its brightness.

The brightness of the light source is a light output that refers to this direction, divided into small (elementary) solid angle near this direction and the projection area of the source on the plane perpendicular to the axis of observation. In other words, this is the ratio of the light force that emitted by the surface area to its projection on a plane perpendicular to the axis of observation.

$$
\mathrm{J}(\theta)=\frac{\mathrm{dI}(\theta)}{\mathrm{d} \sigma \cos \theta}
$$

In additive RGB system that is used in most modern computers brightness of a minimum element of "pixel" is defined as

$$
\mathrm{J}=0,222 \cdot \mathrm{R}+0,707 \cdot \mathrm{G}+0,071 \cdot \mathrm{B}
$$

where $\mathrm{R}$ - the percentage of red at the point (measured from 0 to 255), G and B - green and blue respectively.

Thus, experimental procedure consisted in fixing of brightness of water distribution, transfer images to a computer appearance, layout with different brightness determining the brightness 
of each element in the graphics program Adobe Photoshop, determining the difference between the brightness of the source material and the shaded part.

We used three types of fibres with properties that are shown in Table 1. These materials are used as bandages and wound dressings. The sorption processes are very important for them, because they are usually used as materials for medical textiles and wound dressings.

Table 1

The density of the material and the bulk density $\gamma 5$ main types of textile yarns

\begin{tabular}{lcc} 
Thread type & Density of material, $\mathbf{~} \mathbf{m g} \mathbf{m m}^{\mathbf{3}}$ & Volume $\mathbf{~ m a s s , ~} \mathbf{m g} / \mathbf{m m}^{\mathbf{3}}$ \\
\hline Cotton yarn & 1,5 & 0,9 \\
Wool & 1,3 & 0,7 \\
Viscose yarn & 1,5 & 0,8
\end{tabular}

The experiment was conducted in such a way. The vessel with coloured water was placed under the lab bench (Fig. 1). The water level was at the table level. Textile yarn dropped to one side of the vessel. The main part of thread are laid down on the bench and pressed against the glass plate. The length of the free end of the thread was $20-25 \mathrm{~mm}$. The length of the end submerged in the water, was $10-15 \mathrm{~mm}$. Distribution of water conducted due to the diffusion properties of the yarn.

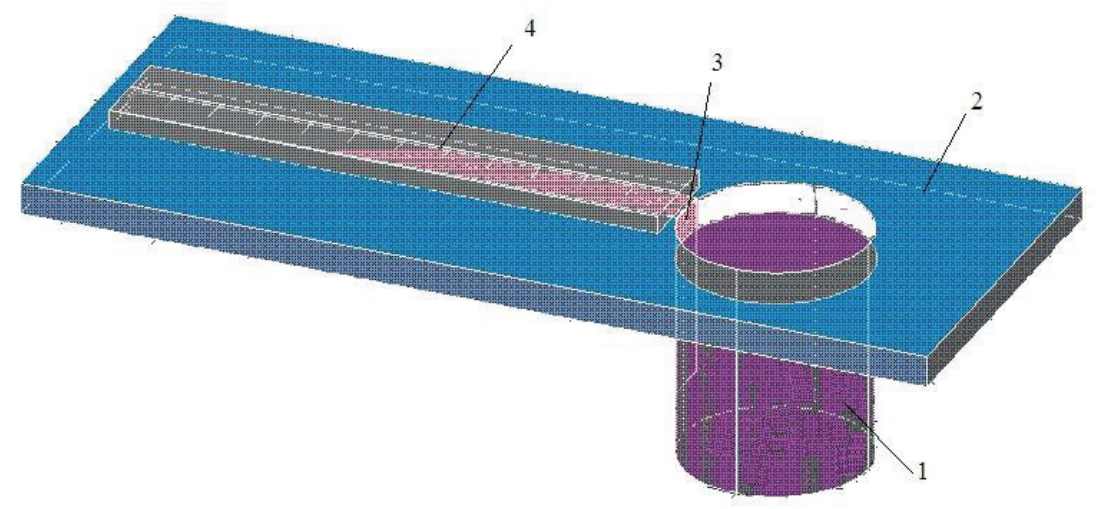

Fig. 1. Scheme of determining the water concentration in the textile material: 1 - vessel with coloured water, 2 - laboratory table, 3 - sample of material, 4 - transparent ruler

Investigated samples were photographed using a web camera through a stereoscopic microscope MBS-10 (Russia). Zone of water distribution was divided into sections. The brightness of each section was determined programmatically using common software Adobe Photoshop CC 2014.

Computer images of painted areas in linear model for different points of time are shown in Fig. 2.

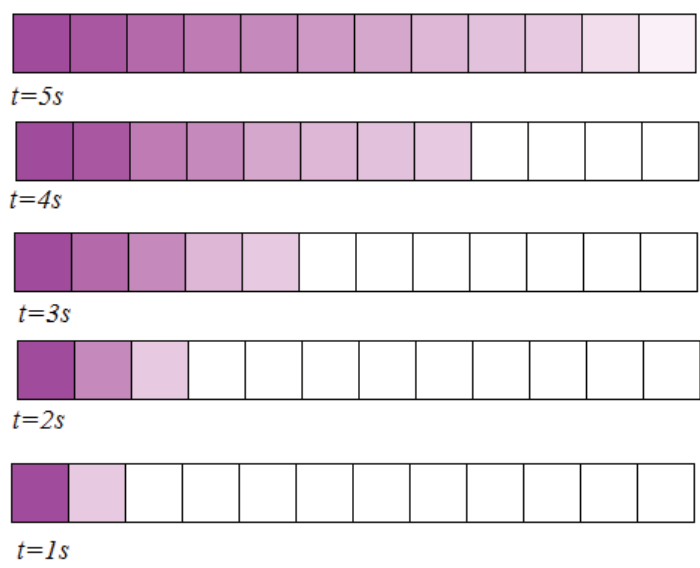

Fig. 2. Brightness change of the water distribution in linear samples, divided into sections 
These results allow us to track a change of the water brightness in linear textile sample in each time period.

\section{Experimental procedures}

The water brightness determines the water concentration in the material. For different points in time change the brightness of the length of the sample is determined by dependence, which is shown in Fig. 3. Here's typical for different samples depending on the example of cotton sample length of $12 \mathrm{~mm}$.

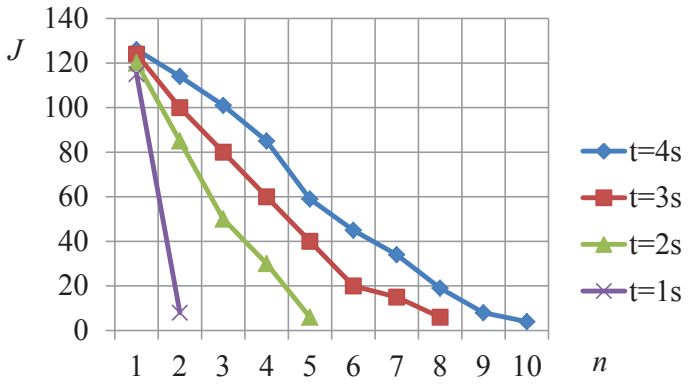

Fig. 3. Brightness change in length for different times depending on the number of section to sample, $\mathrm{n}$ - the section number, which is determined by the brightness, $\mathrm{J}$ - the brightness of the section, $\mathrm{t}$ - time of registration of experimental data (seconds)

At various points of the brightness change by dependences are shown in Fig. 4.

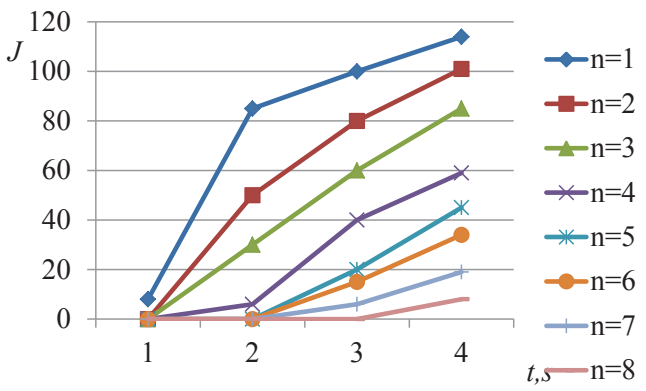

Fig. 4. Brightness change on time for different points of the sample $\mathrm{n}$ - the section number, which is determined by the brightness, $\mathrm{J}$ - the brightness of the section

The zone, which has achieved water in linear sample, is determined by proximity to zero of brightness output colour difference and colour sample wetted zone. Experimental dependence for this zone is shown in Fig. 5. Note that in this case the length of section was $1 \mathrm{~mm}$, i. e. the number of section and distance from the first section numerically are the same.

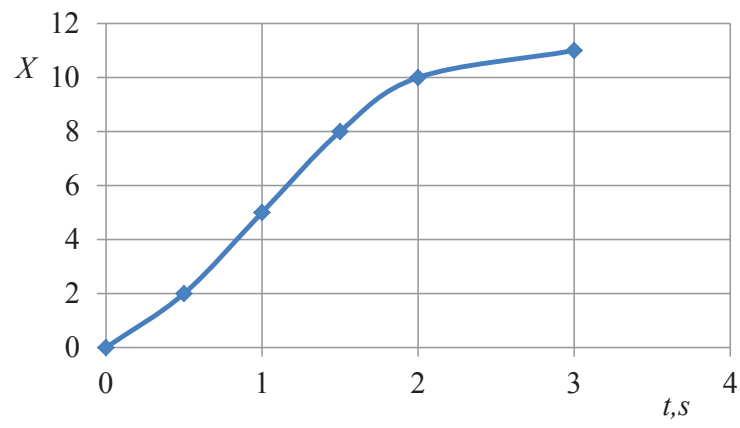

Fig. 5. Change of the boundary of wetted zone in time ( $\mathrm{X}$ - length of wetted, millimeters, $\mathrm{t}$ - time, seconds) 
The hypothesis of brightness compliance of wetted area with the water concentration involves the determining of concentration as the ratio of the current brightness to maximum

$$
\mathrm{u}=\frac{\mathrm{J}}{\mathrm{J}_{\max }} .
$$

We introduce the dimensionless coordinate, that is equal the ratio of the current coordinates to the maximum, which reached water

$$
\bar{x}=\frac{x}{X}
$$

Fig. 6 shows the dependences of determined concentrations in the specific coordinates. It should be noted that the concentration dependences in the specific coordinates are similar.

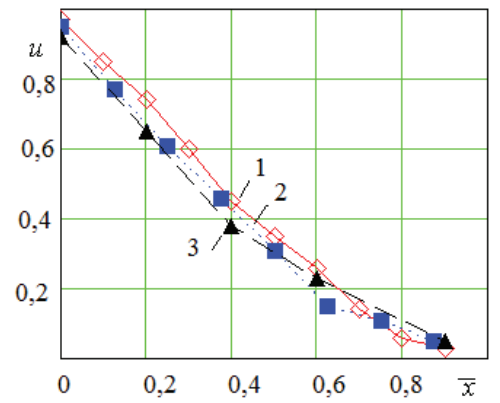

Fig. 6. Dependences of water concentrations in specific coordinates for different points in time $(1-$ dependence after $1 \mathrm{~s}$ of experiment, 2 - dependence after $2 \mathrm{~s}$ of experiment,

$$
3 \text { - dependence after } 3 \mathrm{~s} \text { of experiment) }
$$

Here we received general characteristics. In the process of further analysis we will offer numeric constants, reflecting the spread of water in the linear textile samples.

\section{Results}

Let's analyze the possible form of relationships. The water concentration in a linear sample is determined by differential equation of diffusion.

$$
\frac{\partial \mathrm{U}}{\partial \mathrm{t}}=\frac{\partial}{\partial \mathrm{x}}\left\{\mathrm{D}(\mathrm{U}, \mathrm{x}) \frac{\partial \mathrm{U}}{\partial \mathrm{x}}\right\}
$$

where D - diffusion coefficient, which in common case depends on the water concentration.

In the first approximation, we assume relatively constant diffusion coefficient. Then the equation changes in the concentration may be written as

$$
\frac{\partial U}{\partial t}=D \frac{\partial^{2} U}{\partial x^{2}}
$$

The dependence of the concentration in the specific coordinates will be sought in the form of exponential function.

Let us analyze the dependence shown in Fig. 2, 4. Fig. 2 shows that the concentration on left border closes to unity, concentration on the right boundary asymptotically approaching zero.

Mathematically this dependence can be described by the exponential function of the form

$$
\mathrm{U}=\mathrm{e}^{-\mathrm{A} \cdot \mathrm{x}},
$$

where the coefficient A depends on time. 
Substituting in the diffusion equation, we get

$$
\frac{d A}{d t} A \cdot e^{-A x}=A^{2} \cdot e^{-A x}
$$

The functions of the right and left are reduced, which means that the proposed function corresponds to the differential equation and matched correctly. For unknown factor remains the equation $\frac{\mathrm{dA}}{\mathrm{dt}}=\mathrm{A}$, that is solved by separation of variables in the form

$$
\mathrm{A}=\mathrm{C} \cdot \mathrm{e}^{-\mathrm{t}} \text {. }
$$

Let's leave until the time dependence. We look for different points in time factor value as a fixed value.

To use the method of minimum squares let's use the function

$$
\mathrm{U}=\mathrm{B} \cdot \mathrm{e}^{-\mathrm{A} \cdot \overline{\mathrm{x}}} \text {. }
$$

Logarithm of both sides of the equation gives the result We obtain

$$
\ln \mathrm{U}=\ln \mathrm{B}-\mathrm{A} \cdot \overline{\mathrm{X}} \text {. }
$$

Thus the resulting linear expression with unknown coefficients and a number of experimental points is obtained. So we can use the method of least squares

Solution system provides value

$$
\mathrm{u} \approx 1,02 \cdot \mathrm{e}^{-2,71 \cdot \overline{\mathrm{x}}} \approx \mathrm{e}^{-2,71 \cdot \overline{\mathrm{x}}} .
$$

That is shown in Fig. 7.

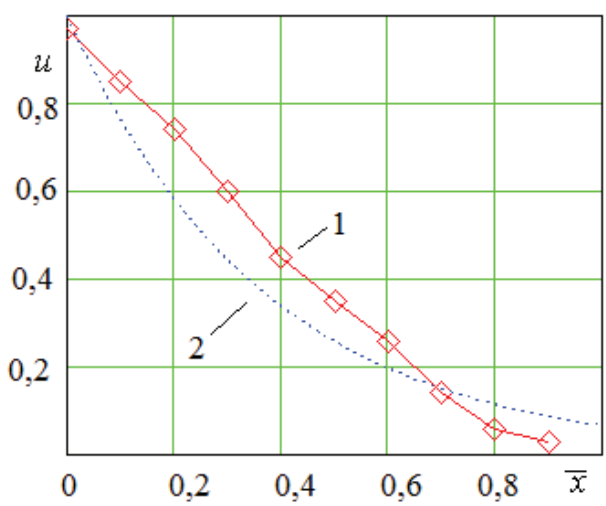

Fig. 7. Water distribution approximation $(1$ - experimental data, 2 - data of the proposed model)

For other points in time the value is determined in the same way. Defining the expectation and variance of the results for cotton fiber can write

$$
\mathrm{u} \approx \mathrm{e}^{-2,7( \pm 0,042) \frac{\mathrm{x}}{\mathrm{x}}} .
$$

Water distribution area $\mathrm{X}$ is a function of time (Fig. 4)

Analyzing dependence, we can offer a function 


$$
\mathrm{X}=\mathrm{C}_{1}\left(1-\mathrm{e}^{-\mathrm{C}_{2} \cdot \mathrm{t}}\right)
$$

For ease of use the method of least squares rewrite function in the form

$$
\mathrm{X}=\mathrm{X}_{\max }-\mathrm{C}_{1} \cdot \mathrm{e}^{-\mathrm{C}_{2} \cdot \mathrm{t}}
$$

where $\mathrm{X}_{\max }$ - maximum of water distribution area.

Then for using the method of least squares transform into such a dependency

$$
\ln \left(X_{\max }-X\right)=\ln C_{1}-C_{2} \cdot t
$$

The simulation results give

$$
\mathrm{X}=12\left(1-\mathrm{e}^{-0,75 \cdot t}\right)
$$

That is shown in Fig. 8.

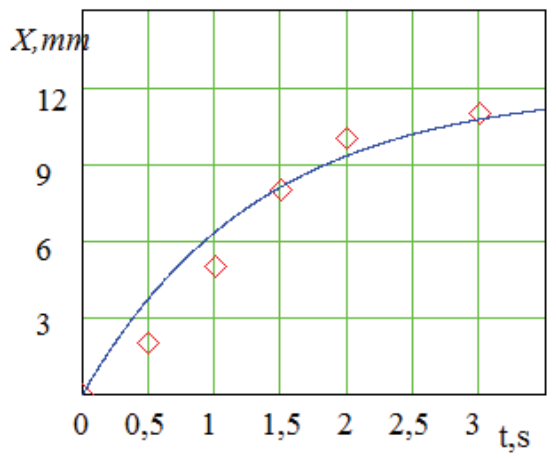

Fig. 8. Changing the boundary of wetted zone

The obtained experimental dependence for the sample, so looks like

$$
\mathrm{u} \approx \mathrm{e}^{0,225 \frac{\mathrm{x}}{1-\mathrm{e}^{-0,75 \cdot \mathrm{t}}}} .
$$

In the future, we will work with the transitional water accumulation functions. At this stage, we will assume the function of the growth moistened zone boundaries in linear way. In this case, this function will look like

$$
X=\left\{\begin{array}{l}
C_{1}+C_{2} \cdot t, t \leq t_{k r} \\
X_{\max }, t>t_{k r} .
\end{array}\right.
$$

Determination of regression coefficients for cotton sample gives function

$$
\mathrm{X} \approx 4,6 \mathrm{t},
$$

that graphically depicted in Fig. 9.

So, in this case we can write the function changes in concentration as

$$
u=e^{-0,59 \frac{x}{t}}
$$

The experiments for another samples allowed to find other similar expressions for other materials and recommend them as typical material constants (Table 2). 


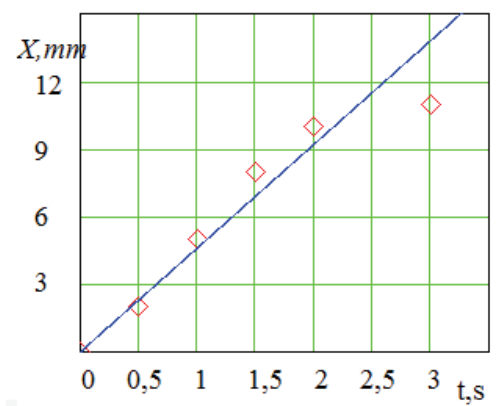

Fig. 9. Linear function of boundary change

Table 2

Factors of water distribution in various materials

\begin{tabular}{cc}
\hline Material & Distribution ratio, s/mm \\
\hline Cotton & 0,59 \\
Wool & 0,51 \\
Viscose & 0,67
\end{tabular}

So, in general terms for water distribution we can use dependency

$$
\mathrm{u}=\mathrm{e}^{-\mathrm{a} \frac{\mathrm{x}}{\mathrm{t}}}
$$

where a is constant characterizing sorption intensity in linear model of material.

\section{Conclusions}

Thus, in this study the general form of empirical distribution functions in linear liquid samples is proved. The concentration dependence of the water from the coordinates and time is proposed. Constants that describe the intensity of a linear sorption of the sample of material are defined. The results can be used in predicting the water distribution and process modeling in discrete material structure.

Empirical function of changes the water concentration in a linear pattern allows to predict the state of the wetted textile materials during their operation, as well as predict their durability. Thus, the aim of the study is performed. Further researches is planned to consider the dependence of the constants on the structure and composition of the material.

The resulting dependence of moisture spread in textiles can be used in the development and operation of medical textiles and wound dressings. Thus, it becomes possible to predict the time of water passage through the material and also to estimate the water concentration in a time in any point of material.

\section{References}

[1] Chen, Q., Miao, X., Mao, H., Ma, P., Jiang, G. (2016). The Comfort Properties of Two Differential-Shrinkage Polyester Warp Knitted Fabrics. Autex Research Journal, 16 (2). doi: 10.1515/aut-2015-0034

[2] Ozturk, M. K., Nergis, B., Candan, C. (2010). A study of wicking properties of cotton-acrylic yarns and knitted fabrics. Textile Research Journal, 81 (3), 324-328. doi: 10.1177/0040517510383611

[3] Afzal, A., Hussain, T., Malik, M. H., Rasheed, A., Ahmad, S., Basit, A., Nazir, A. (2014). Investigation and modeling of air permeability of Cotton/Polyester blended double layer interlock knitted fabrics. Fibers and Polymers, 15 (7), 1539-1547. doi: 10.1007/s12221-014-1539-3

[4] Prakash, C., Ramakrishnan, G., Koushik, C. V. (2013). Effect of blend proportion on moisture management characteristics of bamboo/cotton knitted fabrics. Journal of the Textile Institute, 104 (12), 1320-1326. doi: 10.1080/00405000.2013.800378

[5] Su, C.-I. (2006). Optimum Drafting Conditions of Non-circular Polyester and Cotton Blend Yarns. Textile Research Journal, 76 (6), 441-447. doi:10.1177/0040517506064254 
[6] Su, C.-I., Fang, J.-X., Chen, X.-H., Wu, W.-Y. (2007). Moisture Absorption and Release of Profiled Polyester and Cotton Composite Knitted Fabrics. Textile Research Journal, 77 (10), 764-769. doi: 10.1177/0040517507080696

[7] Das, B., Das, A., Kothari, V., Fanguiero, R., Araujo, M. D. (2009). Moisture Flow through Blended Fabrics - Effect of Hydrophilicity. Journal of Engineered Fibers and Fabrics, 4 (4), 20-28.

[8] Wang, J.-H., Yasuda, H. (1991). Dynamic Water Vapor and Heat Transport Through Layered Fabrics: Part I: Effect of Surface Modification. Textile Research Journal, 61 (1), 10-20. doi: $10.1177 / 004051759106100102$

[9] Adler, M. M., Walsh, W. K. (1984). Mechanisms of Transient Moisture Transport Between Fabrics. Textile Research Journal, 54 (5), 334-343. doi:10.1177/004051758405400510

[10] Suprun, N. (2003). Dynamics of moisture vapour and liquid water transfer through composite textile structures. International Journal of Clothing Science and Technology, 15 (3/4), 218-223. doi: 10.1108/09556220310478314

[11] Riabchykov, N., Vlasenko, V., Arabuli, S. (2011). Linear mathematical model of water uptake perpendicular to fabric plane. Vlakna a textil, 2 (18), 24-29.

[12] Ilhan Ozen (2012). Multi-layered Breathable Fabric Structures with Enhanced Water Resistance. Journal of Engineered Fibers and Fabrics, 7, 63-69.

[13] Adanur, S., Sears, W. (1995). Handbook of Industrial Textiles. USA: Technomic Publishing Corp. Inc., 832.

[14] Li, Y. (2001). The science of clothing comfort. Textile Progress, 31 (1-2), 1-135. doi: 10.1080/00405160108688951

[15] Rief, S., Glatt, E., Laourine, E., Aibibu, D., Cherif, C., Wiegmann, A. (2011). Modeling and cfd-simulation of woven textiles to determine permeability and retention properties. Autex Research Journal, $11(3), 78-83$.

[16] Laourin, E., Cherif, C. (2011). Characterization of barrier properties of woven fabrics for surgical protective textiles. Autex Textile Research Journal, 11 (2), 31-36.

[17] Mishra, R., Kremenakova, D., Behera, B. K., Militky, J. (2011). Structural design engineering of woven fabric by soft computing. Autex Research Journal, 11 (2), 37-41.

[18] Schutskaya, A., Suprun, N. (2015). Discrete two-dimensional model of moisture spreading in textile materials. Vlákna a textil. Textile, 34, 12-17.

[19] Schutskaya, A., Suprun, N. (2016). Discrete three-dimensional model of moisture spreading in textile materials Vlákna a textile, 23 (2), 15-22.

[20] ASTM E96 (1996). E96M - 10, Standard Test Methods for Water Vapor Transmission of Materials.

[21] ASTM E96 (2009). Cup Method Water Vapor Permeability Testing Lab think Instruments Co., Ltd. 